\title{
Neuronal Processes Involved in Subjective Feeling Emergence: Oscillatory Activity During an Emotional Monitoring Task
}

\author{
Elise S. Dan Glauser · Klaus R. Scherer
}

Accepted: 11 February 2008/Published online: 14 March 2008

(C) Springer Science+Business Media, LLC 2008

\begin{abstract}
Subjective feeling, defined as the conscious experience of emotion and measured by self-report, is generally used as a manipulation check in studying emotional processes, rather than being the primary focus of research. In this paper, we report a first investigation into the processes involved in the emergence of a subjective feeling. We hypothesized that the oscillatory brain activity presumed to underlie the emergence of a subjective feeling can be measured by electroencephalographic (EEG) frequency band activity, similar to what has been shown in the literature for the conscious representation of objects. Emotional reactions were induced in participants using static visual stimuli. Episodes for which participants reported a subjective feeling were compared to those that did not lead to a conscious emotional experience, in order to identify potential differences between these two kinds of reactions at the oscillatory level. Discrete wavelet transforms of the EEG signal in gamma $(31-63 \mathrm{~Hz})$ and beta (15-31 Hz) bands showed significant differences between these two types of reactions. In addition, whereas beta band activities were widely distributed, differences in gamma band activity were predominantly observed in the frontal and prefrontal regions. The results are interpreted and discussed in terms of the complexity of the processes required to perform the affective monitoring task. It is suggested that future work on coherent mental representation of multimodal reaction patterns leading to the
\end{abstract}

\section{E. S. Dan Glauser ( $\square)$}

Faculty of Psychology, Unimail, University of Geneva,

40 Bdv du Pont d'Arves, 1205 Geneva, Switzerland

e-mail: elise.dan@pse.unige.ch

K. R. Scherer

Swiss Center for Affective Sciences, University of Geneva,

Geneva, Switzerland emergence of conscious emotional experience should include modifications in the time window examined and an extension of the frequency range to be considered.

Keywords Emotion - Subjective feeling ·

Oscillatory activity $\cdot$ Monitoring $\cdot$ Emotional experience

\section{Introduction}

Subjective feeling can be regarded as the conscious, experiential component of emotion. In Scherer's [1, 2] component process model, this subjective feeling component is thought to reflect changes occurring in other emotional components, such as physiological changes, action tendencies, and vocal and facial expressivity. These emotional responses are further thought to be directly driven by outcomes of the cognitive appraisal process, which is generated by the detection of relevant stimuli [3]. Numerous studies have focused on emotional stimulus processing, but subjective feeling, assessed through verbal report, has generally only been used for manipulation checks. However, conscious subjective experience, as a key component of emotion, is worthy of investigation in its own right.

This is a daunting task, given the complexity of the underlying processes. A vast literature has proposed widely varying definitions of consciousness and its biological roots [4-6]. Previous work seems to suggest that, in many respects, consciousness of external objects is comparable to consciousness of emotional experience. Indeed, both forms of consciousness can be regarded as mental representations $[4,7,8]$, symbolizing external objects or situations in the mind. Moreover, both aim at linking or "binding" the different features of the object or situation in order to 
create a coherent percept or experience. In the case of an object, coherence consists of linking features such as shape, color, or size. In the case of subjective feeling, coherence consists of linking features of situation appraisal, including memory traces, with other components of emotion such as physiological arousal, motor expression, and action tendencies.

One particularly relevant line of research on the emergence of representations in the mind has been pursued by Bertrand and Tallon-Baudry [9] and Tallon-Baudry and Bertrand [10] with respect to object consciousness. They showed that induced oscillatory activity in the gamma band $(30-80 \mathrm{~Hz})$ can be detected differentially when an individual becomes aware of an object's shape. One can hypothesize that the emergent consciousness of a feeling (coherent representation of emotion components) is generated by the same processes, that is through a distinctive oscillatory pattern. In previous research, oscillatory activity has also been reported not to have a unique function, but to serve as an operator, permitting integrative functions to take place [11], and thus serving as a communication code relating activity of different structures [12]. Hence, oscillatory activity seems to be linked to two key features of subjective feeling: its conscious aspect and its integrative aspect; the latter, in the case of emotion, consisting of the integration of changes occurring in other emotional components. The investigation of oscillatory activity during reported subjective feeling may thus contribute to an understanding of the emergence of conscious emotion representation.

In most organisms, rhythmic phenomena at the cerebral level reflect communication between several neuronal assemblies discharging simultaneously [13]. Those simultaneous discharges trigger oscillations at different frequencies, which can be separately analyzed. Prior research has shown that different frequency bands are sensitive to different tasks. In particular, increases in oscillations in gamma $(30-80 \mathrm{~Hz})$ and beta bands $(12-30 \mathrm{~Hz})$ have been reported during complex cognitive functions (mostly attention, learning, and memory, [14, 15]). Gamma oscillations have been implicated in the creation of representation and consciousness emergence (see also [16], and beta oscillations have been indirectly implicated in feature binding [17]. Focusing on gamma and beta bands seems therefore a promising way to tap subjective feeling emergence.

This study is based on the idea that confronting people with emotional stimuli does not necessarily trigger a subjective feeling. We propose to tackle the following question: Are there some detectable patterns in the processing of emotional stimuli that can predict the emergence of emotional experience? We addressed this question by investigating the differences in neural processing between stimulus appraisal episodes that result in the reporting of a subjective feeling, and those that do not.

Research on the experience of emotion has mainly investigated the brain regions involved using various kinds of brain imaging methods [18-21], which offer the unique advantage of high precision in localizing activity. However, the many different processes underlying subjective feeling, such as perception, memory, and language, involve numerous regions. Hence, this approach does not permit one to disentangle the contributions of each region to the process. Although oscillation measurements using electroencephalographic (EEG) methodology present less precise cortical localization than other brain imaging methods, they offer more accurate information on the temporal development of activities while allowing some degree of localization; and are thus best suited to address our research question.

In this study, we attempt to identify specific neurooscillatory activities that are present when people are about to experience a conscious emotion. We hypothesized that at the time of emergence of a subjective feeling (as reported by the participants), oscillatory activity must be of different intensity compared with situations in which the stimulation does not result in such emergence. Higher coefficients were expected for episodes in which subjective feelings are reported, particularly in the gamma band (in accordance with research on the emergence of object consciousness). Exploratory analyses were conducted for basic topographical localization of intensity differences. From reports in the literature, we expected that oscillatory distinctiveness might be reliably observed in the induced oscillatory pattern (stimulus dependent, but not stimulus locked, see [22, 23]). The particularity of the research is the focusing on time periods that are highly variable with respect to the stimulus onsets but strictly locked to the subjective feeling reports.

\section{Methods}

\section{Participants}

Fifty-two female students participated in the study. Exclusion criteria were pregnancy, drug consumption, expertise in psychology (psychology students), severe medical and psychiatric antecedents, and age above 42 years. One participant did not complete the experiment, three procedures were stopped for technical reasons, and four files were excluded from analyses because of low signal-to-noise ratio. The data of 44 participants were therefore included in the study.

The mean age of the participants was 24.8 years (range $18-42 ; S D=4.7)$. All participants were right handed, 
scoring between +22 and +100 on the Handedness Edinburg Inventory [24], with a mean of $+83.49(S D=19)$, and all had normal or corrected-to-normal vision. Additionally, participants were tested and controlled for anxiety and anger (state and trait, with the help of the STAI-S and STAI-T, as well as the STAXI-S and the STAXI-T $[25,26])$. The questionnaires about trait were undertaken a few days before the experiment, whereas the STAI-S and STAXI-S were undertaken before and after the main experiment. Participants were also controlled for difficulty in identifying and reporting personal emotional experience. This latter test was achieved with the help of an alexithymia questionnaire (Toronto Alexithymia Scale, TAS-20 [27]) and with the private self-consciousness score as assessed by the Self-Consciousness Scale [28].

Participants were informed about the experiment and the kind of stimuli they were going to be presented with. All gave informed consent prior to the experiment and were free to abandon it at any time. All participants were paid.

\section{Stimuli}

A database of 480 pictures was used for this experiment. In a previous experiment, all pictures were pretested for valence level. We considered three types of valence: negative, positive, and neutral. Pictures represented daily life scenes, fear-inducing animals, and landscapes. We selected pictures by maximizing the valence differences between the groups of pictures (judged on a scale going from highly negative, 0; to highly positive, 100). Extreme negatives and positives pictures were selected (negative: mean score of $27, \mathrm{SD}=9.1$; positive: mean score of 92 , $\mathrm{SD}=4)$. Selected neutral pictures were rated with middle values (mean score of $53, \mathrm{SD}=5$ ). Additionally, the arousal differences were minimized: Pictures were chosen in such a fashion that arousal scores were situated at the middle level for the three categories of pictures. Lowlevel parameters of the picture (e.g., luminosity histogram shapes, high versus low spectral frequency components of the visual images) were controlled for and did not differ between image categories. Each participant was shown 280 pictures from this database (semi-randomized assignment). Focusing mainly on negative stimulation because of better efficacy of negative affect induction, we showed participants 160 images of negative valence, 60 images of positive valence, and 60 images of neutral valence. Sixty additional images, shown to be of neutral valence in pretests, were used for testing the influence of motor preparation (see the "Procedure" section). Pictures were presented on a 43-cm computer screen at a distance of about $80 \mathrm{~cm}$ from the participant. Each picture was presented for $6 \mathrm{~s}$ with a 3-s stimulus interval.
Procedure

The experiment started with a questionnaire on the participants' state of health and current emotional status. Participants were then informed about the different recordings, and electrodes were then attached.

Participants were installed in a Faraday cage with dimmed light. Instructions were given on the screen before starting the experiment. Most of the experiment consisted of the presentation of the 280 images in succession. The participants performed an affective monitoring task and were instructed to monitor their affective state and to report immediately (via mouse click) when they were affected or touched by the presented image. If the image, even an emotional one, did not trigger any subjective feeling, participants were instructed not to answer. Mouse click was performed with right hand and index finger, and not counterbalanced to avoid biases emerging from a higher difficulty of the task if performed using unusual mouse settings (left hand or other fingers). In addition, supplementary neutral images were presented at the same pace as in the main part of the experiment, and participants reported via mouse click whether the picture represented indoor or outdoor scenes. This motor control procedure (MCP) allowed us to isolate the cortical motor characteristics of a mouse click when the participant was confronted with a picture. At the end of the session, we explained the goal of the experiment to the participants.

\section{Data Acquisition}

$\mathrm{Ag}-\mathrm{AgCl}$ electrodes were fixed on the participants with a Neuroscan (Compumedics ${ }^{\circledR}$ ) quick cap system (62 electrodes), according to the international extended 10-20 system. Electrode impedances were lowered (majority below $5 \mathrm{k} \Omega$, exceptional recordings of electrodes below $30 \mathrm{k} \Omega$ ) with Quick Gel filling (Neuromedics ${ }^{\circledR}$ ), a unilateral mastoid reference electrode was used (counterbalanced). Eye blinks were controlled with vertical electrooculogram electrodes and their artifact reduced by using the default algorithm of blink reduction of Neuroscan (Compumedics ${ }^{\circledR}$ ) with a $10 \%$ threshold. Heart rate, finger temperature, and electromyography on two facial sites were additionally recorded (these measurements will not be reported). The EEG 62-channel data were acquired with Neuroscan (Compumedics ${ }^{\circledR}$ ) at a rate of $500 \mathrm{~Hz}$. The signal was neither low nor high-pass filtered. Data files of the 44 participants were cleaned offline for ocular and movement artifact (trials with amplitudes greater than the value of $105 \mu \mathrm{V}$ were rejected). About $69 \%$ of the trials were retained. Trials were divided in two categories: those having generated a response from the participant (subjective feeling present, SFP, about 120 trials) and those not having generated a subjective 
feeling (no response, subjective feeling absent, SFA, about 75 trials). To be analyzed, each of the trials was trimmed (epoching). SFP trials were epoched $922 \mathrm{~ms}$ before and $100 \mathrm{~ms}$ after the response marker. Epoching was performed on SFA trials by matching one to one each SFP response delay from image onset and considering it a "response" to lock the epoching. SFA and SFP trials were matched according to their valence for each participant. Because the number of SFP trials was generally greater than the number of SFA trials (ratio of about 1.6), each SFA trial was taken into account once for matching, and the remaining match procedure was performed on a randomized selection (of about 45 trials) re-drawn from the entire pool of SFA trials. This procedure was also performed on trials of the MCP with and without response.

\section{Wavelet Analyses}

To extract the targeted frequency information, we transformed the trials before averaging because induced frequency bands were mainly investigated (for a detailed comparison of evoked and induced oscillations and the cancellation of induced activity by premature averaging, see [10, 29]). A wavelet transform was applied to each trial using a B-spline mother wave (Bs3.7) with the S+Wavelets module ( $\mathrm{S}+$, version 2.0.2). For each electrode, the absolute values of the wavelet coefficients were displayed for each trial on the gamma band (about 31-63 Hz) and the beta band (about 15-31 Hz). Vectors were then averaged according to the participant, the frequency band, and the type of trial (SFP versus SFA). About $100 \mathrm{~ms}$ of each extremity of the time windows under consideration were dropped to cope with the boundary problem [30]. The remaining $832 \mathrm{~ms}$ of the trials were grouped into 13 time intervals. Nine topographical regions (Fig. 1) have been delineated by cluster analyses of the activity of the 62 channels (and isolation of medoïds) during the first second following image onset. These topographical regions were selected to investigate cortical localization differences.

\section{Statistical Analyses}

The data follow a within-subject factor design with three independent variables: Time (13) $\times$ Response (SFP versus SFA) $\times$ Region (9) separately for gamma and beta band oscillations. Dependent variable was the gamma wavelet coefficients and the beta wavelet coefficients, respectively. As the main question concerned the variation of differences between SFP and SFA trials before the time at which a subjective feeling emerges, Time $\times$ Response was investigated first with the coefficients averaged over the 62 electrodes. Each region was then tested separately by considering the coefficients averaged over the six or seven

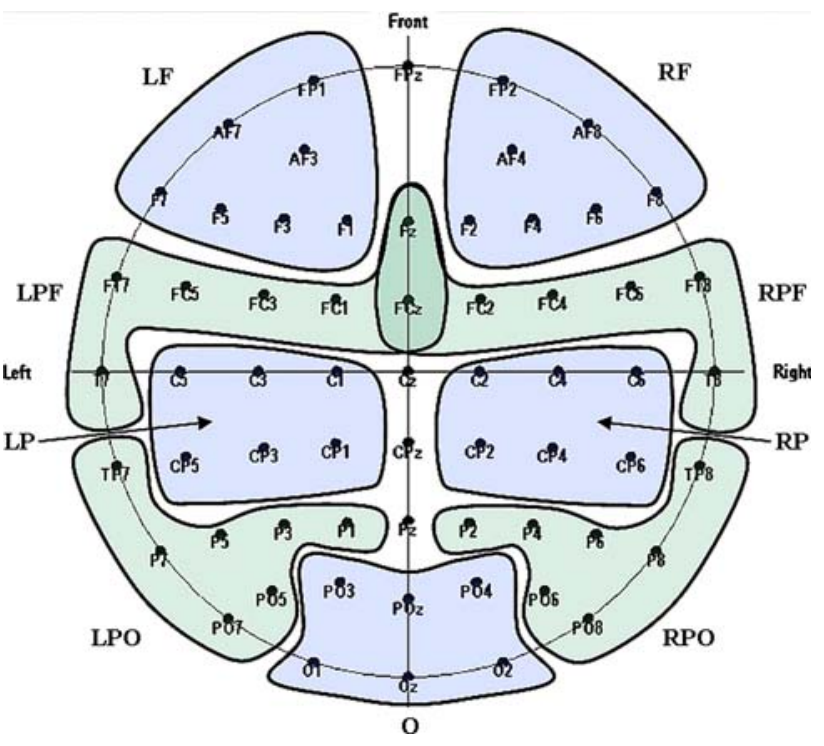

Fig. 1 Electrodes grouped by similar oscillatory activity. LF, left frontal; RF, right frontal; LPF, left prefrontal; RPF, right prefrontal; LP, left parietal; RP, right parietal; LPO, left parieto-occipital; RPO, right parieto-occipital; $\mathrm{O}$, occipital. Illustration from Cartool software (3.22 G, Denis Brunet 1996-2005, Brainmapping, University of Geneva)

electrodes of a particular region. The regions were tested independently to determine at which time points the potential differences between SFA and SFP trials were localized; this, independently of a comparative linkage between coefficients values of all the tested regions.

Motor control procedure trials were also tested according to the same design and used as a control for interpretation of the differences found in the central analysis.

\section{Results}

All results were computed using Greenhouse-Geisser corrections, even if original degrees of freedom are reported for greater clarity. All $P$-values are two-tailed. Partial $\eta$ squares are reported for comparison of effect size $\left(\eta_{\mathrm{p}}^{2}\right)$.

\section{Behavioral Responses}

On average, participants responded to $62.17 \%$ of the trials $(S D=17.4)$. The mean time needed to provide an answer, corresponding to the emergence of subjective feeling, was $2,011 \mathrm{~ms}(S D=647) ; 80 \%$ of the trials were responded with reaction times greater than $800 \mathrm{~ms}$.

\section{Gamma Band Oscillations}

A repeated measures analysis of variance (ANOVA; Time $\times$ Response) performed on wavelet coefficients of 
gamma band activity yielded a significant interaction effect, $F_{(12,516)}=7.44, P<0.01, \eta_{\mathrm{p}}^{2}=0.15$. Tukey HSD post hoc tests $(P<0.05)$ showed significant differences between SFP and SFA trials at several points. For those points, contrasts demonstrated stronger oscillatory activity in trials free of subjective feeling experience than in trials leading to subjective experience of emotion. Results are shown in Fig. 2.

The same analysis performed on MCP trials showed no significant difference between trials containing a motor preparation and the execution of a motor response, and trials free of motor preparation/response, $F_{(12,516)}=2.08$, $P=n s$.

A general time effect has been observed, $F_{(12,516)}=$ 156.1, $P<0.01, \eta_{\mathrm{p}}^{2}=0.78$, showing that in the time window under consideration, oscillatory activity in the gamma band presented an ascending slope. Bonferroni contrasts showed a significant linear increase every $128 \mathrm{~ms}$.

Repeated measures ANOVAs performed for the nine isolated regions of the scalp, $F_{(12,516)}=$ between 2.57 and 4.24, $P<0.05$, showed that the differences between SFA and SFP trials were present only for the left frontal (LF), right frontal (RF), left prefrontal (LPF), and right prefrontal (RPF) regions (see Fig. 1). The time window in which these differences were significant (tested with Tukey HSD post hoc test with a $P$-value $<0.05$ ) ranged from 826 to $250 \mathrm{~ms}$ before the response for the LF region, from 634 to $314 \mathrm{~ms}$ before the response for the RF region, and from 698 to $250 \mathrm{~ms}$ before the response for the LPF and RPF regions.

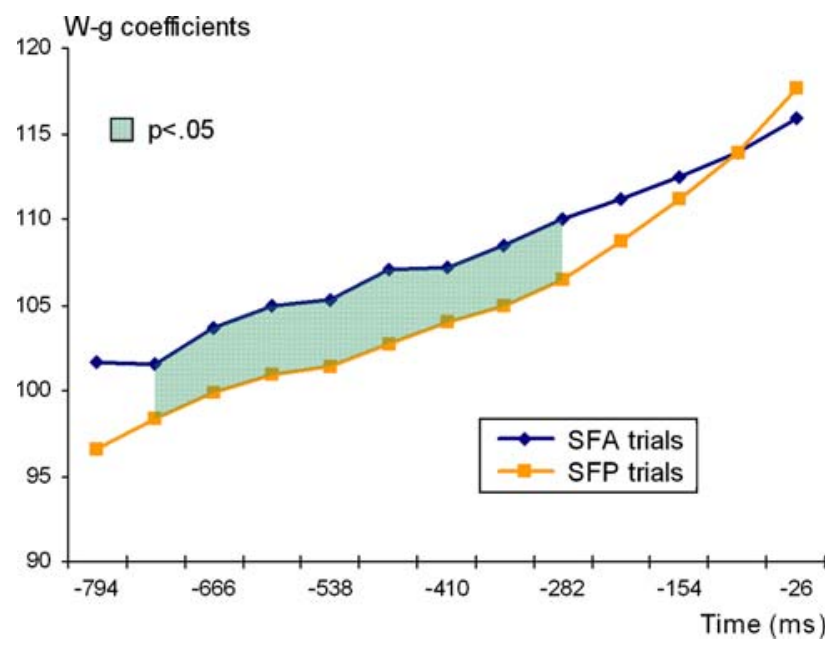

Fig. 2 Temporal development of the wavelet coefficients linked to oscillatory gamma activity (W-g) $800 \mathrm{~ms}$ before subjective feeling report (orange line, SFP trials) and for the same temporal windows following images that did not lead to subjective feeling (blue line, SFA trials). The green shadowed surface represents significant contrasts (Tukey HSD post hoc tests at $P<0.05$ level) between the presence and absence of subjective feeling at a given time point
No difference was noted between the three levels of valence (positive, negative, and neutral trials, $F_{(24,1032)}=$ $1.41, P=n s)$.

Note also that analyses of the first $800 \mathrm{~ms}$ after stimulus onset show no effect of response in the gamma band, $(\mathrm{F}(1,43)=0.064, P=\mathrm{ns})$, showing that not differences are present between SFA and SFP trials just after the stimulation.

\section{Beta Band Oscillations}

Similar to that performed for gamma oscillations, a repeated measures ANOVA (Time $\times$ Response) performed on wavelet coefficients of beta band activity yielded a significant interaction effect, $F_{(12,516)}=2.40, \quad P<0.05$, $\eta_{\mathrm{p}}^{2}=0.05$. Tukey HSD post hoc tests $(P<0.05)$ showed significant differences between SFP and SFA trials between 794 and $154 \mathrm{~ms}$ before the response, again indicating stronger oscillatory activity in trials free of subjective feeling experience than in trials leading to a subjective experience of emotion. Results are illustrated Fig. 3.

Motor control procedure trials also presented a similar interaction (Time $\times$ Response, $F_{(12,516)}=4.19, P<0.01$ ). Contrasts showed that differences between responsive and non-responsive trials presented the inverse oscillatory pattern compared with the SFA and SFP comparison, this time showing higher coefficients for responsive trials than for unresponsive trials.

A general time effect was observed, $F_{(12,516)}=70.58$, $P<0.01, \eta_{\mathrm{p}}^{2}=0.62$, showing that in the time window

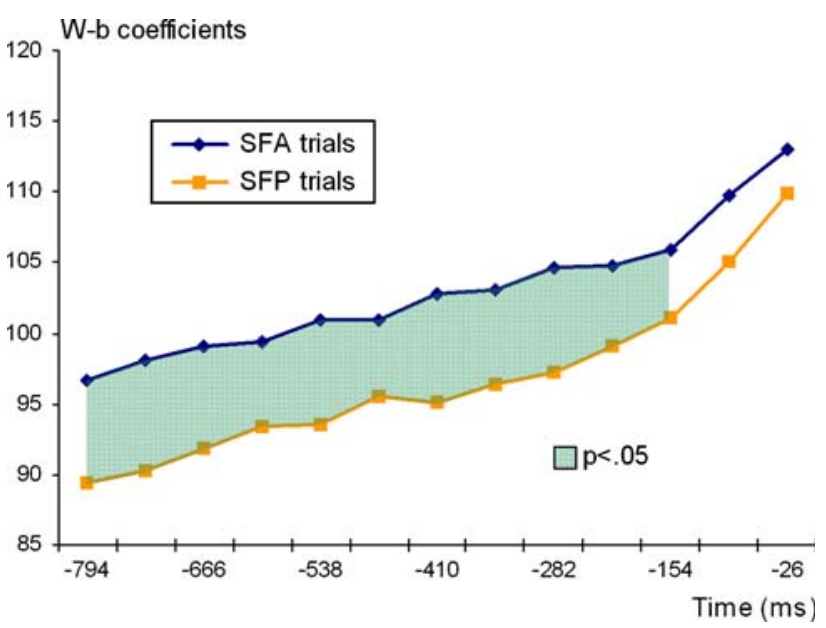

Fig. 3 Temporal development of the wavelet coefficients linked to oscillatory beta activity (W-b) $800 \mathrm{~ms}$ before subjective feeling report (orange line, SFP trials) and for the same temporal windows following images that did not lead to subjective feeling (blue line, SFA trials). The green shadowed surface represents significant contrasts (Tukey HSD post hoc tests at $P<0.05$ level) between the presence and absence of subjective feeling at a given time point 
under consideration, oscillatory activity in the beta band presented an ascending slope. Bonferroni contrasts showed a significant linear increase every $192 \mathrm{~ms}$.

Repeated measures ANOVAs performed for the nine isolated regions of the scalp did not show particular differences between them. The general activation presented in Fig. 3 is reproduced on the whole surface of the scalp.

Beta band analyses with the valence factor showed that the results were valid for emotional pictures (positive, $F_{(1,43)}=13.76, P<0.01$; negative, $F_{(1,43)}=16.49, P<$ 0.01 ), whereas neutral trials did not show differences between SFP and SFA trials, $F_{(1,43)}=1.6, P=\mathrm{ns}$.

Note also that, similarly to the results found for the gamma band, analyses of the first $800 \mathrm{~ms}$ after stimulus onset show no effect of response in the beta band $(\mathrm{F}(1,43)=0.25, P=\mathrm{ns})$, showing that not differences are present between SFA and SFP trials.

\section{Discussion}

In this study, we explored the brain oscillation activity specifically occurring when an individual reports a conscious emotional experience. We examined whether, at the time of emergence of a subjective feeling, oscillatory activity was different compared with situations in which the stimulation did not produce such a report.

Results show that for both gamma and beta frequencies, differences can be systematically observed between stimulus appraisal episodes that do and do not lead to subjectively felt emotion experiences. However, contrary to our hypothesis, more oscillatory activity is present when the task of emotional monitoring is maintained throughout the image presentation without resulting in a subjective feeling report.

None of these differences can be attributed to differences between trials containing a motor preparation and the execution of the response, and trials free of motor activity. In fact, for gamma frequencies, MCP trial analyses do not show any differences. For beta band frequencies, the inverse pattern for MCP trials in comparison with the differences between SFA and SFP trials excludes the contribution of motor preparation and movement execution to the significant differences reported in Fig. 3. Furthermore, these differences are not present in early processing of the stimulation, showing that the differentiation appears only in late processing, beyond the first second of stimulus presentation.

Higher coefficients were expected for SFP trials, particularly in the gamma band. However, contrasts show the inverse result, higher coefficients being found for trials free of subjective feeling. One interpretation of these results is that the oscillatory activity strength that we measured in this study accompanies ongoing emotional monitoring task rather than predicts the emergence of subjective feeling. Given that most of the oscillatory activation we found was roughly located in the frontal cortical region, it could be argued that we monitored the performance of the monitoring task rather than the emergence of conscious subjective feeling (which no longer requires emotional monitoring). In fact, previous research has shown that emotional stimuli can be processed with fast and automatic low-feature analyses (mainly subcortical) [31, 32]. It is thus possible that, at the cortical level, only the aspects related to the complex monitoring task are visible. Conversely, previous (unpublished) data showed that SFP trials correspond to the presentation of stimuli that are relevant to the participant specific sensitivity, which also involved confrontation with more familiar settings. In this experiment, SFP trials may thus induce lower level of oscillatory activity [33] because of higher familiarity with the stimulation proposed, in comparison with unfamiliar scenes (possibly specifically linked to SFA trials), which induce a higher level of oscillatory activity. Globally, our results probably illustrate different processes that need to be further tested concerning subjective monitoring and report and their relationship to the presented stimuli. It may be necessary to examine a larger time window (e.g., up to $2 \mathrm{~s}$ before response) in order to detect the emergence of feeling more precisely. In fact, it is possible that the process involved in the emergence of a subjective feeling is associated with a cortical oscillatory pattern that is identifiable with the present method, but occurs earlier than $1 \mathrm{~s}$ before the response. If this interpretation holds, the SFP trials in the present study only identified participants who had already abandoned the monitoring task and thus show less oscillatory activity during the recorded period.

The results concerning the two specific frequency bands in this research warrants two comments. First, it is obvious that gamma frequency is worth focusing on in research designed to understand conscious processes. Indeed, gamma oscillations are clearly involved in such processes [34, 35]. However, recent studies on slower waves, such as theta $(4-8 \mathrm{~Hz})$, directly implicate such oscillatory activity in emotional processes [36] and may be a promising focus for investigations concerning subjective feeling emergence. Analyses of such waves are currently being performed to explore this hypothesis. Second, preliminary analyses of stimulus valence show a sensitivity of beta waves to valence level that is not reproduced in the results of gamma coefficients. This observation suggests that specific stimulus features may be represented by different frequency bands. Although this research concerns local and discrete oscillatory measurement, it may be considered in light of the hypothesis that Varela et al. [37] applied to phase synchronicity. These authors stated that synchronization 
over multiple frequency bands might be the key to integration of a coherent cognition. A similar hypothesis may be applied in the context of oscillatory measurements and should be tested using an experimental design that takes a more balanced approach to valence differences.

For the two frequency bands examined here, results show an increase in neuronal oscillatory activity over time. Different synchronization slopes have been identified for the two frequency bands. Such slopes and their variations may be of interest in obtaining more variability indices of cortical oscillatory activity. To our knowledge, modulation of the rate at which coefficients vary has however never been systematically investigated or described. For alpha frequency, it has been proposed that coefficient variation may serve as a reset function for neuronal circuits, potentially permitting feature grouping [38]. Slope variation in other frequency bands may have a similar function.

Another interesting result of the present research is the topographical mapping highlighted by differences in the two frequency bands that were tested. Although the testing was performed by contrasting which point in time presented a similar difference as the main result (and same direction), we were able to already identify that for gamma oscillations, differences between SFP and SFA trials were detected in frontal and prefrontal areas. Of note is that the task required in this experiment apparently has an impact on processes occurring in regions that are said to be involved in associative functions and therefore high-order cognitive tasks [39-42]. The coherence process, commonly associated with the creation of subjective feeling experience through the integration of interoceptive and exteroceptive changes that result from an emotional situation [2], may be supported by this localization, highlighting the potential connection between heterogeneous information sources. Strict comparison between region power coefficients will need to be performed in future to confirm this result. Moreover, better localization of activity differences, for example, by computing inverse solutions would higher the discrimination between regions and provide better indications of the neural generators involved in such processing.

More extensive control of confounding components (such as motor activity) in the experimental design should be performed in future studies. For example, this study shows that beta oscillations in SFA and SFP trials present no significant differences for neutral stimulations. Because MCP trials were only neutral trials, future MCPs should take into account the interaction between the emotionality of the picture and the task (involving a motor response). This step will ensure better disentanglement of oscillatory patterns specific to the emergence of a subjective feeling and those that are linked to the unavoidable personal report that is simultaneously obtained from the participant.
Finally, investigation of sensitivity to valence (and other stimulus features) of different frequency bands should be analyzed in the time window for early picture processing. This approach could also be a way to investigate whether conscious emotion consequences of a given stimulation (e.g., emergence of subjective feeling) can be detected in the basic analysis of emotional stimulation (as early as a few hundred milliseconds from image onset).

This research constitutes a first approach to identifying subjectivity within the objective biological process of emotion. The importance of the subjective feeling component in emotion requires deeper investigation, and this task invites widely different research directions. As explored in the present research, analyzing differences between the presence and absence of subjective feeling in similar emotional situations, and taking into account the diversity of confounding elements, may help us to understand how becoming aware of our own emotion is resulting from characteristic brain oscillatory activity.

Acknowledgment This research was supported by FNRS grant 51A240-104897 awarded to K.R. Scherer as part of the National Center of Competence in Research (NCCR) in Affective Sciences, University of Geneva, Geneva, Switzerland. The authors thank Sylvain Delplanque for thorough reading and useful comments on first drafts of the manuscript.

\section{References}

1. Scherer KR. On the nature, function of emotion: a component process approach. In: Scherer KR, Ekman P editors. Approaches to emotion. Hillsdale, NJ: Erlbaum; 1984. p. 293-317.

2. Scherer KR. Feelings integrate the central representation of appraisal-driven response organization in emotion. In: Manstead ASR, Frijda NH, Fischer AH editors. Feelings and emotions: the Amsterdam symposium. Cambridge: Cambridge University Press, 2004. p. 136-55.

3. Scherer KR. Appraisal theory. In: Dalgleish T, Power M editors. Handbook of cognition and emotion. Chichester: Wiley; 1999. p. 637-67.

4. Atkinson AP, Thomas MS, Cleeremans A. Consciousness: mapping the theoretical landscape. Trends Cogn Sci 2000;4(10):372-82.

5. Delacour J. Neurobiology of consciousness: an overview. Behav Brain Res 1997;85(2):127-41.

6. Zeman A. Consciousness. Brain 2001;124:1263-89.

7. Craig AD. Forebrain emotional asymmetry: a neuroanatomical basis? Trends Cogn Sci 2005;9(12):566-71.

8. Taylor JG. The central role of the parietal lobes in consciousness. Conscious Cogn 2001;10:379-417.

9. Bertrand O, Tallon-Baudry C. Oscillatory gamma activity in humans: a possible role for object representation. Int J Psychophysiol 2000;38(3):211-23.

10. Tallon-Baudry C, Bertrand O. Oscillatory gamma activity in humans and its role in object representation. Trends Cogn Sci 1999;3(4):151-62.

11. Basar E, Basar-Eroglu C, Karakas S, Schurmann M. Are cognitive processes manifested in event-related gamma, alpha, theta and delta oscillations in the EEG? Neurosci Lett 1999;259(3): $165-8$. 
12. Keil A, Junghofer M. Macroscopic oscillations in emotional perception: a time-frequency approach to the study of motivated attention. Elec Geodes Inc 2002;6(1):1-3.

13. Axmacher N, Mormann F, Fernandez G, Elger CE, Fell J. Memory formation by neuronal synchronization. Brain Res Rev 2006;52(1):170-82.

14. Basar E, Basar-Eroglu C, Karakas S, Schurmann M. Gamma, alpha, delta, and theta oscillations govern cognitive processes. Int J Psychophysiol 2001;39(2-3):241-8.

15. Tallon-Baudry C, Kreiter A, Bertrand O. Sustained and transient oscillatory responses in the gamma and beta bands in a visual short-term memory task in humans. Vis Neurosci 1999;16(3): 449-59.

16. Llinas R, Ribary U. Coherent $40-\mathrm{Hz}$ oscillation characterizes dream state in humans. Proc Natl Acad Sci USA 1993;90(5): 2078-81.

17. Freedman JE. Neuronal information coding by oscillation phase prediction error: implications for consciousness and control of voluntary function. Med Hypotheses 2006;67(2):287-95.

18. Barrett LF, Mesquita B, Ochsner KN, Gross JJ. The experience of emotion. Annu Rev Psychol 2007;58:373-403.

19. Critchley HD, Wiens S, Rotshtein P, Öhman A, Dolan RJ. Neural systems supporting interoceptive awareness. Nat Neurosci 2004;7(2):189-94.

20. Damasio AR, Grabowski TJ, Bechara A, Damasio H, Ponto LLB, Parvizi J, et al. Subcortical and cortical brain activity during the feeling of self-generated emotion. Nat Neurosci 2000;3(10): 1049-55.

21. Heilman KM. The neurobiology of emotional experience. J Neuropsychiatry Clin Neurosci 1997;9(3):439-48.

22. Herrmann CS, Grigutsch M, Busch NA. EEG oscillations and wavelet analysis. In: Handy TC editor. Event-related potentials: a method handbook. Cambridge, MA: MIT Press; 2005. p. 229-59.

23. Infantosi AF, Miranda de Sa AM. A coherence-based technique for separating phase-locked from non-phase-locked power spectrum estimates during intermittent stimulation. J Neurosci Methods 2006;156(1-2):267-74.

24. Oldfield RC. The assessment and analysis of handedness: the Edinburgh inventory. Neuropsychologia 1971;9(1):97-113.

25. Spielberger CD. Manual for the state-trait anxiety inventory (STAI). PaloAlto, CA: Consulting Psychologists Press; 1983.

26. Spielberger CD. State-trait anger expression inventory. Professional manual. Odessa: Psychological Assessment Resources; 1988.
27. Taylor JG, Ryan D, Bagby RM. Toward the development of a new self-report alexithymia scale. Psychother Psychosom 1985;44:191-9.

28. Fenigstein A, Scheier MF, Buss AH. Public and private selfconsciousness: assessment and theory. J Consult Clin Psychol 1975;43(4):522-7.

29. Frund I, Schadow J, Busch NA, Korner U, Herrmann CS. Evoked gamma oscillations in human scalp EEG are test-retest reliable. Clin Neurophysiol 2007;118(1):221-7.

30. Cai W, Wang J. Adaptive multiresolution collocation methods for initial boundary value problems of nonlinear PDEs. SIAM J Numer Anal 1996;33(3):937-70.

31. LeDoux JE. Emotion circuits in the brain. Annu Rev Neurosci 2000;23:155-84.

32. Morris JS, Ohman A, Dolan RJ. A subcortical pathway to the right amygdala mediating "unseen" fear. Proc Natl Acad Sci USA 1999;96(4):1680-5.

33. Gruber T, Muller MM. Oscillatory brain activity in the human EEG during indirect and direct memory tasks. Brain Res 2006;1097(1):194-204.

34. Bojak I, Liley DTJ. Self-organized $40 \mathrm{~Hz}$ synchronization in a physiological theory of EEG. Neurocomputing 2007;70:2085-90.

35. Melloni L, Molina C, Pena M, Torres D, Singer W, Rodriguez E. Synchronization of neural activity across cortical areas correlates with conscious perception. J Neurosci 2007;27(11):2858-65.

36. Sammler D, Grigutsch M, Fritz T, Koelsch S. Music and emotion: electrophysiological correlates of the processing of pleasant and unpleasant music. Psychophysiology 2007;44(2):293-304.

37. Varela FJ, Lachaux J-P, Rodriguez E, Martinerie J. The brainweb: phase synchronization and large-scale integration. Nat Neurosci Rev 2001;2:229-39.

38. Pfurtscheller G, Stancak A Jr, Neuper C. Event-related synchronization (ERS) in the alpha band-an electrophysiological correlate of cortical idling: a review. Int J Psychophysiol 1996;24(1-2):39-46.

39. Duncan J, Owen AM. Common regions of the human frontal lobe recruited by diverse cognitive demands. Trends Neurosci 2000; 23(10):475-83.

40. Fuster JM. Executive frontal functions. Exp Brain Res 2000; 133(1):66-70.

41. Smith EE, Jonides J. Storage and executive processes in the frontal lobes. Science 1999;283(5408):1657-61.

42. Stuss DT, Alexander MP. Executive functions and the frontal lobes: a conceptual view. Psychol Res 2000;63(3-4):289-98. 